\title{
ANÁLISIS DE INSTRUMENTO PARA EVALUACIÓN DEL DESEMPEÑO EN ACTIVIDADES DE LA VIDA DIARIA INSTRUMENTALES LAWTON Y BRODY
}

\author{
ANALYSIS OF THE LAWTON AND BRODY INSTRUMENTAL ACTIVITIES OF DAILY
} LIVING ASSESSMENT INSTRUMENT.

\section{Karla Hernández $\mathrm{P}^{1}$. y Vivian Neumann $\mathrm{C}^{2}$}

\begin{abstract}
Resumen
El propósito fue analizar el Índice de Lawton y Brody (ILB), determinando si los factores género, desempeño previo y nivel socioeconómico afectan las conclusiones relacionadas con la dependencia en AVDI.

La metodología es analítica, exploratoria y transversal. En primera instancia se aplicó una encuesta de percepción de ILB a 22 terapeutas ocupacionales (TTOO) pertenecientes a servicios públicos de Valparaíso, Viña del Mar, Concón y Quilpué. En segunda instancia se aplicó ILB más un cuestionario complementario a 36 usuarios del Centro Comunitario de Rehabilitación de Miraflores, Instituto de Neurorehabilitación Luis Krebs y Centro de Rehabilitación Integral Siloe; paralelamente, el terapeuta ocupacional (TO) completó una encuesta con su impresión sobre la capacidad de realizar las AVDI de sus usuarios, para determinar diferencias significativas entre ambos. Resultados: Los resultados indican que los TO están en desacuerdo con las características del instrumento. En los resultados de su aplicación, el ILB permite identificar el nivel de dependencia en AVDI en los ítems que evalúa. Se condujó que el factor género no es determinante de la dependencia, pero sí es determinante el factor socioeconómico; el nivel de desempeño previo tiene una correlación positiva con el desempeño actual de las personas.

Conclusiones: La percepción de los TO está en desacuerdo con ILB. Los resultados de la aplicación de ILB y la contrastación con la impresión del TO tratante permite confirmar que efectivamente el instrumento logra el objetivo para el cual fue diseñado, destacando que el género no es un factor determinante en la realización de AVDI, mientras el factor socioeconómico es considerado como relevante para su realización.
\end{abstract}

\section{Palabras clave:}

terapia ocupacional, Índice Lawton y Brody, actividades de la vida diaria instrumental, dependencia.

\begin{abstract}
Analysis of the Lawton and Brody instrumental activities of daily living assessment instrument.

The aim was to analyze the Lawton and Brody index ( $L B I)$, to determine if factors such as gender, previous performance and socioeconomic level affect the conclusions related to the IADL dependency.

The methodology is analytic, exploratory and cross-sectional. An opinion survey about IADL was applied to 22 OTs belonging to public services in Valparaíso, Viña del Mar, Concón and Quilpué. The LBI plus a 36-question complementary questionnaire were applied to Rehabilitation Community Center in Miraflores, Luis Krebs Neuro-rehabilitation Institute and Comprehensive Rehabilitation Center. Besides, the OTs filled in a survey about his views on the users' abilities to perform IADL in order to establish meaningful differences between both. Results: The results show that occupational therapists don't agree with the instrument features. In the results application, the LBI allows to identify the dependency level in IADL in the assessed items. It was shown that the gender factor is not decisive in dependency, however, the socio-economic factor is decisive; the level of previous performance has a positive correlation with the people's current performance. Conclusions: The OT's perception shows disagreement with the BLI. The results of the application of LBI and the contrast with the OTS opinion allows to confirm that the instrument actually fulfills the objective for which it was designed, pointing out that gender is not a decisive factor in IADL while socio-economic factor is considered relevant in its application.
\end{abstract}

1 Karla Hernández Perez, Terapeuta Ocupacional, Magister en Terapia Ocupacional mención salud física. Jefe de Carrera Terapia Ocupacional Universidad Santo Tomás Sede Viña del Mar. kahernandez@santotomas.cl

2 Vivian Neumann Collyer, Terapeuta Ocupacional, Magister en Dirección y Liderazgo para la Gestión Educacional. Directora Escuela de Terapia Ocupacional, Universidad Santo Tomas Sede Viña del Mar. vivianneumann@santotomas.cl 


\section{Keywords:}

occupational therapy, Lawton and Brody index, instrumental activities of daily living, dependency.

Fecha de recepción: 19/07/2016.

Fecha de aceptación: 21/10/2016.

\section{INTRODUCCIÓN}

Las actividades que realizamos los seres humanos cotidianamente, como asearnos, vestirnos o desplazarnos de un lugar a otro, entre otras, han sido desde los orígenes de la terapia ocupacional objeto de su interés y atención constante. Si bien han estado relacionadas con otras disciplinas, ya en el año 1950 se publica la primera escala de evaluación de independencia en actividades de la vida diaria, elaborada por terapeutas ocupacionales (Moruno, 2006). En la actualidad, tanto las actividades de la vida diaria básicas como instrumentales están definidas en el Marco para el Trabajo de la Terapia Ocupacional de la Asociación Americana de Terapia Ocupacional, publicado en 2008, en donde se establecen ocho áreas ocupacionales que deben ser contempladas al momento de determinar el nivel de participación de una persona en su contexto.

Las AVD se relacionan con la supervivencia del ser humano (comida, vestido, higiene) y de este hecho deriva su repetición diaria; cumplen una función social importante ya que participan en los procesos de socialización a lo largo del desarrollo evolutivo de cada individuo, y su adquisición nos permite insertarnos en el mundo social y cultural al que pertenecemos (Moruro, 2006).

La Organización Mundial de la Salud (OMS) ha reconocido que la salud pude ser afectada por la inhabilidad de llevar a cabo actividades y participar en situaciones de vida, causadas por barreras ambientales, así como por problemas que existen en las estructuras y funciones corporales, lo cual queda establecido en la Clasificación Internacional del Funcionamiento, de la Discapacidad y de la Salud CIF (OMS, 2001).

Por lo tanto, las AVD son uno de los aspectos de mayor relevancia para el ser humano y a la par, de la intervención del terapeuta ocupacional en muchos campos de práctica, en la medida que posibilitan el desempeño independiente y autónomo de actividades cotidianas que contribuyen al bienestar y a la libertad de acción y expresión, así como el desempeño independiente en éstas es un predictor del funcionamiento cognitivo en adultos mayores, (Burton, 2009; Gazquez, 2008; Gold, 2012).
Las actividades instrumentales de la vida diaria (AIVD) son más complejas que las actividades básicas de la vida diaria, y su realización permite que una persona pueda ser independiente dentro de una comunidad, es decir, son actividades que posibilitan la relación con el entorno (Jiménez, 2012). Además, son actividades multipasos y pueden clasificarse en tres grupos: actividades de uso de medios, actividades orientadas al ocio y actividades de trabajo doméstico (Polonio, 2010). Según la Asociación Americana de Terapia Ocupacional, éstas dependen de factores culturales y sociales estableciendo las siguientes actividades: cuidado de los otros, cuidado de mascotas, facilitar la crianza de los niños, gestión de la comunicación, movilidad en la comunidad, uso de la gestión financiera, gestión y mantenimiento de la salud, establecimiento y gestión del hogar, preparación de la comida y limpieza, mantenimiento de la seguridad y responder a la emergencia, y compras (Avila, 2010).

Para la evaluación de éstas existen diversos instrumentos y listas de chequeo, entre los cuales podemos mencionar el Cuestionario VIDA, Inventario de tareas rutinarias (RPI-2), Bels, Inventario de destrezas adaptativas (CALS), Kels y el Índice Lawton y Brody (Martin, 2006; Moruno, 2006; Martin, 2012), los cuales por lo general valoran si una persona es dependiente o independiente. En Chile se utiliza fundamentalmente el Índice de Lawton y Brody (Philadelphia Geriatric Center) publicado en 1969 y traducido al español en 1993 (Lawton, 1969). La puntación final es la suma del valor de todas las respuestas y oscila entre 0 (máxima dependencia) y 8 puntos (independencia total). La información se obtiene preguntando directamente al individuo o a su cuidador principal. El tiempo medio requerido para su realización es de 4 minutos; cualquier miembro del equipo puede aplicar esta escala, pero debe estar motivado y entrenado. La escala de Lawton y Brody es uno de los instrumentos de medición de AVDI más utilizado por los terapeutas ocupacionales (Martin, 2006; Moruno, 2006). Sus mayores limitantes son las variables 
que se estudian, ya que pueden estar influidas por aspectos culturales y del entorno. Las actividades instrumentales son además difíciles de valorar en pacientes institucionalizados por las limitaciones impuestas por el entorno social propio del centro. No todas las personas requieren la misma habilidad ante el mismo grado de complejidad y la puntuación de diversos parámetros de la escala puede reflejar ciertas situaciones sociales, más que el verdadero grado de capacidad del individuo. Se considera una escala más apropiada para las mujeres (muchas de las actividades que mide la escala han sido realizadas tradicionalmente por ellas) pero también se recomienda su aplicación a los hombres, aunque todavía están pendientes de identificar aquellas actividades instrumentales realizadas por ellos según los patrones sociales (Graf, 2013; Trigas, 2011; Zavala, 2011).

Por lo tanto, este instrumento arroja resultados que se pueden ver influidos por el desempeño previo de la persona y por variables de género, socioculturales y/o económicas, ya que no se considera que algunos ítems pueden ser realizados por un tercero, así como tampoco han sido adaptados al siglo XXI.

A partir de lo anterior, en la presente investigación se realizó el análisis del instrumento Lawton y Brody, considerando la percepción de los terapeutas ocupacionales que utilizan el instrumento actualmente y un análisis estadístico en relación a la concordancia de los resultados obtenidos en usuarios y la impresión del TO tratante.

\section{MÉTOdo}

La metodología de la presente investigación se enmarcó en un diseño no experimental, descriptivo, correlacional, exploratorio y transversal. Este estudio se realizó en dos etapas, inicialmente se identificó la percepción de terapeutas ocupacionales sobre el instrumento Lawton y Brody, a través de una encuesta de percepción de 11 preguntas, sometida a validación por juicio de expertos, a una población intencionada de 44 terapeutas ocupacionales que trabajan en los servicios públicos de las ciudades de Valparaíso, Viña del Mar, Quilpué y Concón, durante los meses de junio y agosto del año 2014, obteniéndose una muestra de 22 personas que participaron voluntariamente.

En una segunda etapa se identificaron los niveles de dependencia/independencia de la población en estudio, utilizando el Índice Lawton y Brody y la impresión del TO tratante por medio de una encuesta diseñada para dicho propósito. La muestra fue no probabilística en un estudio poblacional, donde se seleccionaron y participaron voluntariamente con firma de consentimiento informado 36 personas adultas de ambos sexos entre 50 y 75 años, sin daño cognitivo conocido, que acudieron a recibir atención ambulatoria en las unidades de terapia ocupacional del Centro Comunitario de Rehabilitación Miraflores, Instituto de Neurorehabilitación Luis Krebs y el Centro de Rehabilitación Integral Siloe, todos ubicados en la comuna de Viña del Mar, entre los meses de junio y septiembre del año 2014.

Paralelo a la aplicación del ILB, se hizo un cuestionario complementario a la misma muestra que consideró aspectos relacionados con el desempeño previo, género y nivel socioeconómico.

Los datos fueron recopilados por las investigadoras e investigadoras auxiliares, quienes acudieron directamente a los centros ya mencionados durante el citado periodo de estudio donde procedieron a aplicar los instrumentos.

Los datos obtenidos fueron procesados con el programa SPSS versión 22.0.

\section{Resultados}

Con respecto a la percepción de los terapeutas ocupacionales sobre el ILB, en 9 de las preguntas los encuestados están en desacuerdo o muy en desacuerdo con el Índice Lawton y Brody (ILB), estando de acuerdo en que el instrumento logra medir nivel de independencia en AVDI y que tiene un sistema de fácil puntuación. El $100 \%$ de los encuestados está en desacuerdo con que el ILB considera las características culturales de la persona; un 54,5\% está en desacuerdo con que el ILB considera los motivos por los cuales la persona no realiza una actividad contenida en éste; el 63,6\% de los encuestados está en desacuerdo con que el ILB mide el desempeño en las AVDI consideradas en el Marco de Trabajo de la Terapia Ocupacional de la AOTA; un $68,2 \%$ de los encuestados está en desacuerdo con que el ILB utiliza rangos de puntuación que permiten identificar el nivel de asistencia requerida; un $63,6 \%$ de los encuestados está en desacuerdo con que las preguntas del ILB son atingentes a la realidad actual; y el 90,9\% de los encuestados está en desacuerdo con que el ILB como instrumento de evaluación de AVDI cumple las expectativas de los terapeutas ocupacionales en relación a sus resultados (Tabla 1 ). 


\begin{tabular}{|c|c|c|c|c|c|c|c|c|}
\hline & & 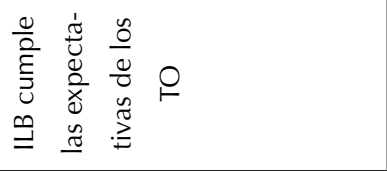 & $N$ & $\stackrel{\infty}{ }$ & $\begin{array}{l}\tilde{8} \\
\dot{8}\end{array}$ & $\sim$ & 0 & $\bar{\sigma}$ \\
\hline & & 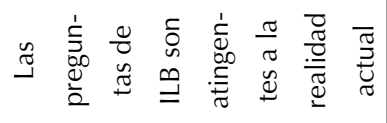 & in & $\sigma$ & $\begin{array}{l}0 \\
\tilde{3}\end{array}$ & $\infty$ & 0 & \begin{tabular}{l}
\multirow{0}{*}{} \\
in
\end{tabular} \\
\hline & & 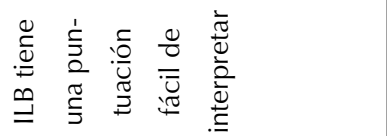 & - & in & $\begin{array}{l}0 \\
\infty^{0} \\
\sim^{2}\end{array}$ & $=$ & $\nabla$ & $\stackrel{t}{\Sigma}$ \\
\hline$\stackrel{\oplus}{=}$ & & 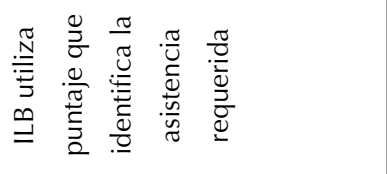 & $\infty$ & $\wedge$ & 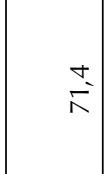 & in & - & $\begin{array}{l}0 \\
\infty^{\circ} \\
\overbrace{}^{2}\end{array}$ \\
\hline 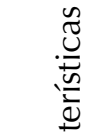 & & 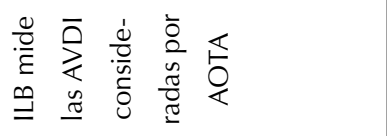 & $\sim$ & $\simeq$ & $\begin{array}{l}0 \\
\tilde{b}^{-}\end{array}$ & $\infty$ & 0 & $\begin{array}{l}+ \\
\stackrel{m}{0}\end{array}$ \\
\hline 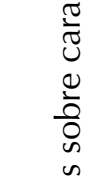 & 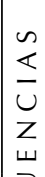 & 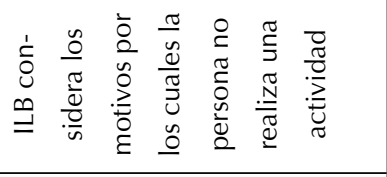 & 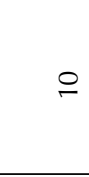 & $\sigma$ & $\begin{array}{l}+ \\
5 \\
\infty \\
\infty\end{array}$ & $m$ & 0 & $\begin{array}{l}\stackrel{0}{m} \\
\dot{m}\end{array}$ \\
\hline 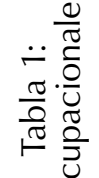 & 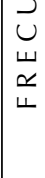 & 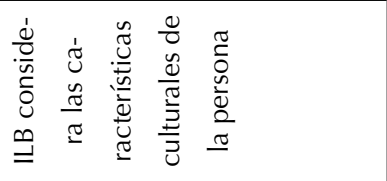 & $\infty$ & \pm & $\begin{array}{l}0 \\
8 \\
0\end{array}$ & 0 & 0 & : \\
\hline 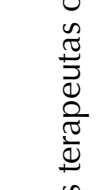 & & 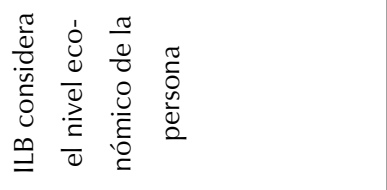 & $\wedge$ & \pm & \begin{tabular}{l}
2 \\
\multirow{2}{\alpha}{}
\end{tabular} & - & 0 & $\stackrel{\llcorner}{\sim}$ \\
\hline $\begin{array}{l}\frac{0}{0} \\
\frac{0}{0} \\
: \frac{0}{U} \\
\frac{0}{0}\end{array}$ & & 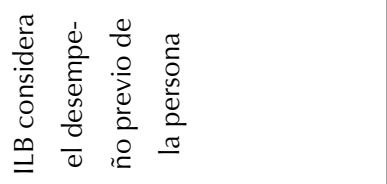 & $\infty$ & $\simeq$ & $\begin{array}{c}n \\
\text { ผू }\end{array}$ & - & 0 & $\stackrel{\infty}{\leftarrow}$ \\
\hline ¿ & & 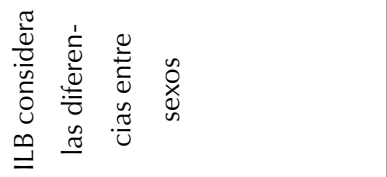 & $\infty$ & $=$ & $\begin{array}{l}\text { Lे } \\
\text { \&े }\end{array}$ & $\sim$ & 0 & $\stackrel{L}{\sigma}$ \\
\hline & & 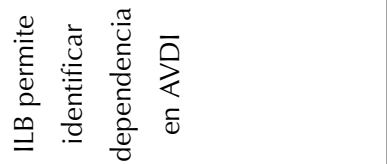 & $N$ & $m$ & $\hat{\tilde{N}}$ & $\underset{\Xi}{ \pm}$ & $m$ & $\stackrel{\Re}{\wedge}$ \\
\hline & 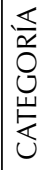 & & 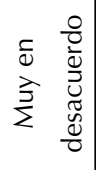 & 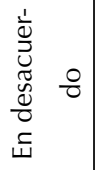 & 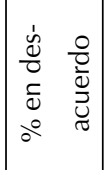 & 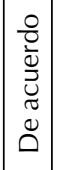 & 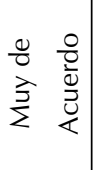 & 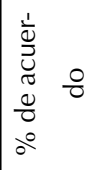 \\
\hline
\end{tabular}


En relación a la identificación de los niveles de dependencia/independencia de la población en estudio, se observó que los criterios de clasificación tanto del ILB como de la impresión del TO tratante son coinci- dentes, existiendo una variabilidad en los puntajes extremos considerando a las personas en un nivel menor o mayor de desempeño (Gráfico 1).

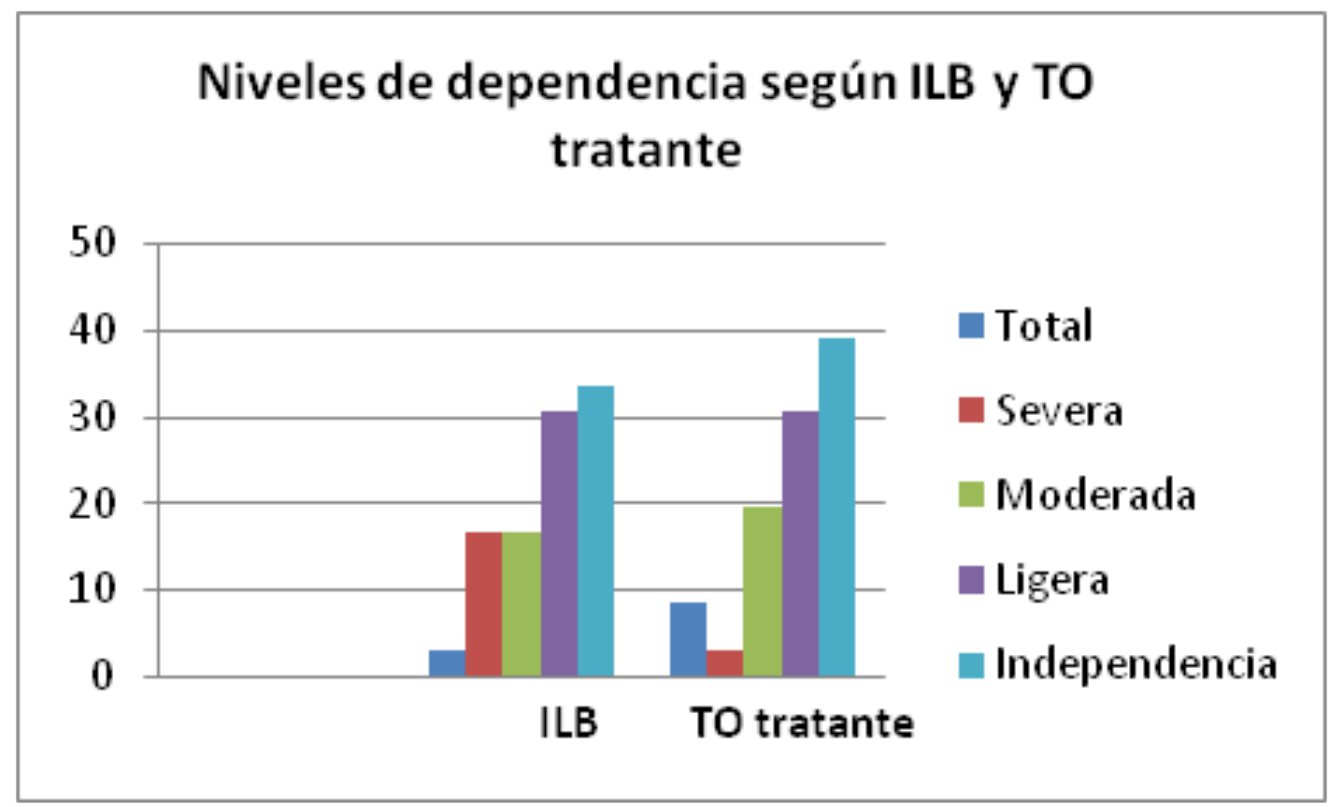

Con respecto al cuestionario complementario, en el desempeño previo de las personas evaluadas se observa que en las actividades de uso de transporte privado y responsabilidad en la medicación existe una muy baja correlación entre ambas variables (podríamos decir que ambas son independientes), sin embargo en el resto de las actividades (uso de red fija, celular, preparación de la comida, cuidado de la casa y manejo de asuntos económicos) es posible apreciar una correlación positiva, es decir existe una relación directamente proporcional entre el desarrollo de esas actividades en el pasado y en el presente, lo que significa que las personas mantienen sus conductas, pues los que realizaban la actividad en el pasado la siguen realizando en el presente y si no las realizaban en el pasado, tampoco las realizan en la actualidad. El desempeño previo no es condicionante del desempeño actual (Tabla 2).
Tabla 2:

Coeficiente de correlación de nivel del desempeño previo de AVDI.

\begin{tabular}{|c|c|}
\hline & Pasado v/s Presente \\
\hline Actividad & Coef. de correlación \\
\hline Uso de red fija & 0,7 \\
\hline Uso de celular & 0,6 \\
\hline Hacer compras & 0,4 \\
\hline Preparar la comida & 0,6 \\
\hline Cuidado de la casa & 0,6 \\
\hline Uso de transporte público & 0,4 \\
\hline Uso de transporte privado & 0,2 \\
\hline Responsabilidad en la Medicación & 0,1 \\
\hline Manejo de asuntos económicos & 0,6 \\
\hline
\end{tabular}


Acerca de la relación de las AVDI con el factor género, ante la pregunta " $i$ Considera que esta actividad es propia de su género?", se aprecia que la mayoría de los encuestados indica que las actividades del ILB no dependen de este factor, donde los porcentajes más bajos que señalan la no dependencia del género son de un $78 \%$ en la actividad "Realizar compras" y un $81 \%$ en la actividad "cuidar la casa". En efecto, podríamos concluir que en un alto porcentaje, la opinión de los encuestados coincide en que la influencia del género en las actividades expuestas anteriormente no es significativa (Tabla 3).

Tabla 3:

Factor género en el desempeño de AVDI.

\begin{tabular}{c|c|c|c|c|c|c|c|c|c|}
\hline Categorias & Uso Red Fija & Uso Celular. & $\begin{array}{c}\text { Realizar las } \\
\text { Compras }\end{array}$ & $\begin{array}{c}\text { Preparar la } \\
\text { Comida }\end{array}$ & Cuidar la Casa & $\begin{array}{c}\text { Uso } \\
\text { Transporte } \\
\text { Público }\end{array}$ & $\begin{array}{c}\text { Uso } \\
\text { Transporte } \\
\text { Prirado }\end{array}$ & $\begin{array}{c}\text { Responsabilid } \\
\text { ad Medicación }\end{array}$ & $\begin{array}{c}\text { Manejo } \\
\text { asuntos } \\
\text { Económicos }\end{array}$ \\
\hline $\mathrm{N}_{0}$ & 32 & 34 & 28 & 31 & 29 & 35 & 33 & 31 & 34 \\
\hline$\%$ & 89 & 94 & 78 & 86 & 81 & 97 & 92 & 89 & 94 \\
\hline $\mathrm{Si}$ & 4 & 2 & 8 & $\mathbf{5}$ & 7 & 1 & 3 & 4 & 2 \\
\hline$\%$ & 11 & 6 & 22 & 14 & 19 & 3 & 8 & 11 & 6 \\
\hline Total & 36 & 36 & 36 & 36 & 36 & 36 & 36 & 35 & 36 \\
\hline
\end{tabular}

Se aprecia que solamente existen dos actividades con un bajo porcentaje de dependencia de los recursos económicos de las personas, que son cuidar la casa y manejar los medicamentos con un 25\% y un 39\% respectivamente; alrededor del $50 \%$ de las personas opina inciertamente sobre la dependencia de los recursos económicos en las actividades del uso del teléfono, ya sea fijo o móvil, y la realización de compras; y sobre el $70 \%$ opina que las actividades de uso de transporte público y privado y el manejo de los asuntos económicos sí dependen de los recursos económicos en su realización (Tabla 4$)$.

Tabla 4: Factor recursos económicos en el desempeño de AVDI.

\begin{tabular}{|c|c|c|c|c|c|c|c|c|c|}
\hline \multirow[t]{2}{*}{ Categorías } & \multirow[t]{2}{*}{ Uso Red Fija } & \multirow[t]{2}{*}{ Uso Celular } & \multirow{2}{*}{$\begin{array}{l}\text { Realizar } \\
\text { Compras }\end{array}$} & \multirow{2}{*}{$\begin{array}{c}\text { Preparar la } \\
\text { Comida }\end{array}$} & \multirow{2}{*}{ Cuidar la Casa } & \multirow{2}{*}{$\begin{array}{c}\text { Uso Transporte } \\
\text { Público }\end{array}$} & \multirow{2}{*}{$\begin{array}{c}\text { Uso Transporte } \\
\text { Privado }\end{array}$} & \multicolumn{2}{|c|}{ Responsabilida $\mid$ Manejo asuntos } \\
\hline & & & & & & & & d Medicación & Economicos \\
\hline $\mathrm{N}_{0}$ & 18 & 17 & 15 & 14 & 27 & 10 & 9 & 22 & 10 \\
\hline$\%$ & 50 & 47 & 42 & 39 & 75 & 28 & 25 & 61 & 28 \\
\hline $\mathrm{Si}$ & 18 & 19 & 21 & 22 & 9 & 26 & 27 & 14 & 26 \\
\hline$\%$ & 50 & 53 & 58 & 61 & 25 & 72 & 75 & 39 & 72 \\
\hline Total & 36 & 36 & 36 & 36 & 36 & 36 & 36 & 36 & 36 \\
\hline
\end{tabular}




\section{DisCUSIÓN}

Según los resultados obtenidos en torno a la percepción de los terapeutas ocupacionales sobre el ILB, la mayoría de los encuestados considera que el instrumento permite medir la dependencia en AVDI, sin embargo consideran que el ILB como instrumento de evaluación de AVDI no cumple las expectativas de los terapeutas ocupacionales en relación a sus resultados, estando en desacuerdo con las características del mismo No obstante, por la falta de un instrumento acorde a las necesidades de la disciplina, sigue siendo utilizado por los profesionales ya que permite identificar si el usuario es en la actualidad dependiente en el desempeño de las AVDI incluidas en el mismo (Moruno, 2006).

En estos resultados se observó que los terapeutas ocupacionales refieren que el instrumento no aborda la totalidad de las AVDI definidas por el marco para el trabajo de la AOTA, sin embargo, algunos terapeutas ocupacionales sí consideran que están incluidas, lo cual genera interrogantes acerca de las actualizaciones de los profesionales sobre las nuevas nomenclaturas que se establecen a nivel internacional (Ávila, 2010).

Las investigadoras creen que la contradicción entre el uso del instrumento y la baja aprobación del mismo se debe a que es el único instrumento accesible existente en Chile. Además, se recomienda su uso por parte de los organismos de Salud, excluyendo a otras pautas disponibles por falta de validación y estandarización de las mismas. Se observa que un porcentaje importante de terapeutas ocupacionales no utiliza ningún instrumento estandarizado y crean herramientas de evaluación en su práctica profesional que no son validadas, por lo tanto no arrojan estadísticas significativas reales de los niveles de dependencia en AVDI y solo sirven en sus lugares de trabajo bajo una perspectiva subjetiva del desempeño ocupacional en esta área.

En torno a los resultados obtenidos en la aplicación del ILB a usuarios de los servicios de terapia ocupacional de los 3 centros en estudio evaluados, en conjunto con la entrevista al TO tratante para identificar su impresión sobre el desempeño en AVDI de los usuarios, los resultados indican que los puntajes otorgados con el ILB en comparación con la impresión del TO tratante son coincidentes en los niveles medios (ligero y moderado), observando una diferencia en los niveles extremos (independencia, severa y total), donde se aprecian más de éstos con el ILB respecto de la impresión del TO tratante. Los especialistas lo consideran un índice con muchas limitaciones, sin embargo en términos generales permite identificar los niveles de dependencia de los usuarios debido a su excelente confiabilidad y validez (Vergara, 2012).

Los terapeutas ocupacionales encuestados manifestaron que el ILB no considera la dimensión género, al igual que los diversos estudios revisados en los cuales se pesquisa como principal limitación el hecho de que se considera una escala más apropiada para las mujeres según los patrones sociales típicos (Tomas, 2003; Trigás, 2011). Sin embargo, el cuestionario complementario mostró que ésta no será relevante a la hora de aplicar la evaluación de independencia en AVDI. Esta diferencia podría deberse a que en el siglo XXI no existen roles masculinos y femeninos marcados relacionados con quehaceres domésticos como en el siglo pasado, por lo que ambos sexos realizan las diferentes actividades evaluadas en igual proporción.

En la mayoría de los evaluados se observó la existencia de una relación de ciertas actividades con el nivel socioeconómico, esto es respecto al acceso que se tiene a herramientas de mayor calidad, comodidad y modernidad según los recursos económicos, por lo que a mayor sean éstos, mayor acceso se tiene a automóviles particulares, celulares con alta tecnología y lavadora, entre otros, por eso este estudio muestra que es importante pesquisar el nivel socioeconómico de las personas para conocer el acceso que éstas tienen a herramientas que le facilitan la ejecución de algunas AVDI.

Acerca del desempeño previo de las actividades existe una correlación positiva con el desempeño actual en las actividades uso del teléfono, hacer compras, cuidar la casa, lavado de ropa, medio de transporte y capacidad de utilizar el dinero, hecho que no se aprecia en preparación de la comida y responsabilidad sobre la medicación, por lo cual no podemos concluir que el desempeño previo tenga un impacto en la ejecución de las AVDI en el presente.

En conclusión, los resultados obtenidos nos permiten identificar que si bien el ILB es un instrumento que permite evaluar el desempeño en AVDI, sus preguntas y sistema de puntación generan desacuerdo en quienes lo utilizan, considerando la antigüedad del instrumento y su poca adecuación al contexto local, lo que hace necesario generar un instrumento actualizado que considere los factores anteriormente descritos. 


\section{REFERENCIAS BIBLIOGRÁFICAS}

Ávila, A., Martínez, R. Matilla, R., Máximo, N., Méndez, B., Talavera, M. y Rivas, N. (2010) Marco de trabajo para Terapia Ocupacional: Dominio y Proceso. Recuperado el 10 de enero de 201. Disponible en http://www.terapia-ocupacional.com/aota2010esp.pdf.

Burton, C., Strauss, E., Bunce, D., Hunter, M. \& Hultsch, D. (2009). Functional Abilities in Older Adults with Mild Cognitive Impairment, Gerontology; 55: 570-581.

Gazquez, J., Rubio, R., Pérez, M. y Lucas, F. (2008). Análisis de los factores predictores de la dependencia funcional en personas mayores. International Journal of Psychology \& Psychological Therapy, 8 (1), $117-126$

Gold, D. (2012). An examination of instrumental activities of daily living assessment in older adults and mild cognitive impairment. Journal of clinical and experimental neuropsychology, 34 (1), 11-34.

Graf, C. (2013). The Lawton instrumental activities of daily living (IADL) scale. Try This: Best practices innursing care in older adults, 23. Recuperado el 13 de enero de 2014. Disponible en consultgerirn. org/uploads/File/trythis/try_this_23.pdf.

Jiménez, P. y López, F. (2012). Valoración de las actividades instrumentales de la vida diaria tras un ictus mediante la escala de Lawton y Brody. Rev Neurol; 55 (6), 337-342.

Lawton, MP. \& Brody, EM. Assessment of older people: selfmonitoring and instrumental activities of daily living. Gerontologist, 179-186. Recuperado el 15 de abril de 2014. Disponible en http://www.eurohex.eu/bibliography/pdf/Lawton_Gerontol_1969-1502121986/ Lawton_Gerontol_1969.pdf.

Martin, I. y Ortiz, I. (2006). Identificación de ítems para la creación de un cuestionario de valoración de actividades instrumentales de la vida diaria en personas mayores. Atención Primaria, 37 (6), 313-318

Martin, I., Quintana, S., Urzay, V., Ganzarain, E., Aguirre, T. \& Pedrero, J. (2012) Fiabilidad del cuestionario VIDA, para valoración de Actividades Instrumentales de la Vida Diaria (AIVD) en personas mayores. Atención Primaria, 44(6), 309-319.

Moruno, P. y Romero, D.M. (2006). Actividades de la vida diaria. En D. Pérez \& D.M. Romero, Herramientas para la evaluación de las actividades de la vida diaria (pp. 77-127). Barcelona: Elsevier.

Organización Mundial de la Salud y Organización Panamericana de la Salud (2001). Clasificación Internacional del Funcionamiento, de la Discapacidad y de la Salud CIF.

Polonio, B. y Romero, D. (2010). Terapia Ocupacional aplicada al daño cerebral adquirido, en Evaluación funcional y ocupacional, (pp. 179-199). Madrid: Médica Panamericana.

Tomas, C. y Zunzunegui, M. (2003). Dependencia evitable para las actividades de la vida diaria: una perspectiva de género. Revista Española de Geriatría y Gerontología, 38 (6) 327-333.
Trigas, M., Ferreira, L. y Meijide, H. (2011). Escalas de valoración funcional en el anciano. Galicia Clínica, 72(1), 11-16.

Vergara, I., Bilbao, A., Oriven, M., García-Gutiérrez, S., Navarro, G. \& Quintana, J. (2012). Validation of the Spanish version of de Lawton IADL scale for its application in elderly people. Health and quality of life outcomes. Recuperado el 5 de septiembre de 2014. Disponible en http://www.hqlo.com/content/10/1/130.

Zavala, M. \& Domínguez, G. (2011). Funcionalidad para la vida diaria en adultos mayores. Revista Médica del IMSS, 49 (6), 585-590. 